\title{
Systematic Review: Depression and Anxiety Prevalence in Bladder Cancer Patients
}

\author{
Liliana Vartolomei $^{\mathrm{a}, \mathrm{b}}$, Matteo Ferro ${ }^{\mathrm{c}}$, Vincenzo Mirone ${ }^{\mathrm{d}}$, Shahrokh F. Shariat ${ }^{\mathrm{a}, \mathrm{e}, \mathrm{f}, \mathrm{g}, *}$ and Mihai Dorin \\ Vartolomei $^{\mathrm{a}, \mathrm{h}}$ \\ ${ }^{a}$ Department of Urology, Medical University of Vienna, Vienna, Austria \\ ${ }^{\mathrm{b}}$ Department of Clinical Psychology, University "Dimitrie Cantemir”, Tirgu Mures, Romania \\ ${ }^{\mathrm{c}}$ Division of Urology, European Institute of Oncology, Milan, Italy \\ ${ }^{\mathrm{d}}$ Department of Neurosciences, Human Reproduction and Odontostomatology, University of Naples, Naples, \\ Italy \\ ${ }^{\mathrm{e}}$ Karl Landsteiner Institute of Urology and Andrology, Vienna, Austria \\ ${ }^{\mathrm{f}}$ Department of Urology, University of Texas Southwestern Medical Center, Dallas, TX, USA \\ ${ }^{\mathrm{g}}$ Department of Urology, Weill Cornell Medical College, New York, NY, USA \\ ${ }^{\mathrm{h}}$ Department of Cell and Molecular Biology, University of Medicine and Pharmacy, Tirgu Mures, Romania
}

\begin{abstract}
.
Background: Depression affects more than 300 million people of all ages worldwide. In patients with cancer the reported prevalence is up to $24 \%$.

Objective: To systematically review the literature to report the prevalence of depression and anxiety among patients with bladder cancer (BC).

Methods: Web of Science, MEDLINE/PubMed, and The Cochrane Library were searched between January and March 2018 using the terms "bladder carcinoma OR bladder cancer AND depression OR anxiety".

Results: Thirteen studies encompassing 1659 patients with BC were included. Six studies assessed depression prior and after treatment at 1, 6 and 12 months. Three were conducted in the US, one each in Turkey, Sweden/Egypt and China. Four studies showed a reduction of depression after radical cystectomy (RC) at 1, 6 and 12 months, respectively. Contrary, two studies showed no significant difference in depression between baseline and follow-up. Four studies investigated anxiety; they reported a slight reduction in anxiety score compared to baseline. Seven additional studies reported the prevalence of depression and anxiety (five studies) among patients with BC at a specific time-point. Studies were conducted in Sweden (2), Italy, Greece, US, China and Spain. Pretreatment depression rates ranged from 5.7 to $23.1 \%$ and post-treatment from 4.7 to $78 \%$. Post-treatment anxiety rates ranged from 12.5 to $71.3 \%$.

Conclusions: The prevalence of reported depression and anxiety among BC patients is high with large geographic heterogeneity. Gender and geriatric specific screening and management for anxiety and depression should be implemented to alleviate suffering.
\end{abstract}

Keywords: Depression, anxiety, bladder cancer, prevalence, systematic review

\section{INTRODUCTION}

${ }^{*}$ Correspondence to: Shahrokh F. Shariat, MD, Department of Urology, Comprehensive Cancer Center, Medical University of Vienna, Vienna General Hospital, Währinger Gürtel 18-20, A1090 Vienna, Austria. Tel.: +43 140400 26150; Fax: +43 140400 23320; E-mail: shahrokh.shariat@meduniwien.ac.at.
Depression affects more than 300 million people of all ages worldwide and less than half are receiving treatment according to World Health Organization (WHO) [1]. Approximately $10 \%$ to $15 \%$ of older adults have clinically significant depressive 
symptoms, even in the absence of major depression [2]. Depression prevalence in cancer patients and survivors [3] ranges from 8 to 24\% [4]. The prevalence was shown to be higher in long-term cancer survivors compared to healthy controls (11.6 vs. 10.2\%) [5].

Depression affects patient's ability to cope with the burden of the illness decreasing the acceptance of treatment, extending hospitalization, reducing quality of life and increasing suicide risk by $50 \%$ for men and 30\% for women [6]. Effective treatments which may be offered for moderate and severe depression include behavioural activation, cognitive behavioural therapy (CBT), and interpersonal psychotherapy (IPT) [7, 8] or antidepressant medication such as selective serotonin reuptake inhibitors (SSRIs) and tricyclic antidepressants (TCAs) [9].

Another major psychological distress of patients with cancer is anxiety. Pretreatment, on-treatment and post-treatment anxiety prevalence's were $19.12 \%$ (CI $17.11 \%$ to $21.30 \%$ ), $26.23 \%$ (CI $22.30 \%$ to $30.56 \%$ ) and $27.09 \%$ (CI $23.10 \%$ to $31.49 \%$ ) in women with ovarian cancer in a meta-analysis that included 24 studies [10]. The prevalence was shown to be higher in long-term cancer survivors than in healthy controls (17.9 vs. $13.9 \%$ ) [5].

Palliative care is now recommended by the American Society of Clinical Oncology (ASCO) as the standard of care for patients with advanced cancer based on increasing evidence of its beneficial impact on quality of life, symptom management, anxiety and depression, caregiver distress and aggressive end-oflife care $[11,12]$.

In patients with prostate cancer ( $\mathrm{PCa}$ ), depression and anxiety rates were shown to be higher than in general population with pretreatment, on-treatment and post-treatment depression prevalence's of $17.27 \%$, $14.70 \%$ and $18.44 \%$, and anxiety prevalence's of $27.04 \%, 15.09 \%$ and $18.49 \%$, respectively [13]. In patients with testicular cancer, anxiety levels were higher than in the general population, while depression levels did not differ [14]. Moreover, it was shown that the risk of suicide in patients with urological cancers was 5-fold higher than that in the general population with an incidence of 48/100,000 in bladder, 36/100,000 in kidney, and 52/100,000 in prostate cancer patients according to a population study that included 328,372 patients with urological malignancies [15]. While these rates are indicative, they do not account for regional and stage specific distribution and dynamic changes.

During survivorship, patients with bladder cancer (BC) reported several unmet needs centered around psychological support (i.e., depression, poor body image and sexual dysfunction) and instrumental support (e.g. difficulty adjusting to changes in daily living) and these are varying by age, sex, and treatment choice $[16,17]$. While according to a national database study $8.8 \%$ of BC survivors had major depressive disorder [18].

The aim of our study was to systematically review the literature reporting the prevalence of depression and anxiety among patients with BC.

\section{EVIDENCE ACQUISITION}

\section{Protocol}

The protocol has been registered in the International Prospective Register of Systematic Reviews

Database (PROSPERO: CRD42018086979). Data searches were conducted between January and March 2018. We searched in Web of Science, MEDLINE/PubMed, and The Cochrane Library for articles that met inclusion criteria using association of terms that included bladder carcinoma OR bladder cancer AND depression OR anxiety. No restrictions on publication dates were imposed. To supplement the electronic searches, we also conducted cross searching of references lists of previous reviews, key papers and other relevant articles identified by the electronic search. Two investigators performed the literature search independently and disagreements were resolved by consensus with a third investigator.

\section{Eligibility criteria}

The PICOS (Population, Intervention, Comparator, Outcome and Study design) approach was utilized. Eligibility was according to the Preferred Reporting Items for Systematic Reviews and Meta-analysis (PRISMA) criteria (www.prismastatement.org) [19]. Firstly, studies were considered eligible if they report depression and anxiety $(\mathrm{P})$ prior treatment for bladder cancer (I) and after treatment at specific time points during follow-up (C) to determine if there is a change in depression and anxiety status (O), using validated scales for depression and anxiety (S). Secondly, studies were considered eligible if they reported the specific prevalence of depression and anxiety in patients with $\mathrm{BC}$ at any time during diagnosis, treatment and survivorship. These studies were included to determine prevalence of depression and anxiety within BC patients. Studies published in conference proceedings, 
qualitative research, commentaries and discussions, letters, books, book chapters or research not published in English language were excluded. If patients with $\mathrm{BC}$ were included within an investigation that recruited mixed cancer populations, the study was included if reported data about the patients with BC as a distinct subgroup.

\section{Study selection}

Titles and abstracts were initially assessed for eligibility. If it was possible to confirm that an article met the inclusion criteria from the abstract alone, the full text article was retrieved. If it was clear from the abstract that an article was not eligible, it was rejected immediately. If it was not possible to determine the eligibility of an article from the abstract, the full text article was retrieved. If any key information was missing, we contacted the authors for the missing data. If this was not possible or ineffective, the study was rejected.

\section{Data extraction}

The following specific information relating to data collection and results was extracted individually from each identified article and entered into a predesigned Excel spreadsheet: first author, year and geographical location (country) of data collection; aims and objectives of the investigation; study design; participant inclusion and exclusion criteria; recruitment procedures; sample size; disease stage; age; time since diagnosis; stage of treatment (pretreatment, ontreatment or post-treatment); treatments (TURB or $\mathrm{RC}$ ); depression and anxiety scales utilized; depression prevalence $(\%)$ and anxiety prevalence $(\%)$; and depression and anxiety scores according to utilized scales at baseline and at post treatment follow-up.

\section{RESULTS}

\section{Search results}

The electronic database searches initially yielded 869 results. Of these, 369 were subsequently removed due to duplication. Five hundred abstracts were then retrieved and critically appraised for the inclusion criteria. Of these 13 articles meet the inclusion criteria (Fig. 1).

Six studies [20-25] assessed the depression using validated scales prior to treatment and after treatment at 1,6 or 12 months. Three were conducted in the US, one each in Turkey, Sweden/Egypt and China. The sample sizes of the studies varied widely from 29 to 244 patients. The total sample size across all 6 studies was 520 patients. Data on participant age was reported by 5 of the 6 studies; the mean age across the studies varied from 54.8 to 68.3 years. All studies included patients who underwent $\mathrm{RC}$ and one included also patients with NMIBC (155 pts.) [25]. Three studies used Hospital Anxiety and Depression scale (HADS) [22-24] and Beck's Depression Inventory (BDI) [20]; Basic Symptom Inventory-18 (BSI-18) [21]; Center for Epidemiologic Studies Depression Scale (CES-D) [25] were used in one study each. Two studies showed a reduction of depression after 12 months in patients treated with RC [20, 22], others did not show a significant difference in depression between baseline and followup. In four studies [21, 22, 24, 25] that investigated anxiety; there was a slight reduction in anxiety scores compared to baseline (Table 1).

Other seven studies reported the prevalence of depression among patients with BC [26-32]. Studies were conducted in Sweden (2), Italy, Greece, the US, China and Spain. The sample sizes of the studies varied from 39 to 441 patients. The total sample size across all seven studies was 1139 patients. Data on participant age was reported by 6 of the 7 studies; the mean age across the studies varied from 63.7 to 70.7 years. All studies included patients who underwent $\mathrm{RC}$ and three included also patients with NMIBC (554 patients/48.6\%). Three studies used HADS; two used CES-D; BDI and a well-being questionnaire were used in one study each. Pretreatment depression rates ranged from 5.7 to $23.1 \%$. Post-treatment depression rates ranged from 4.7 to $78 \%$. Two studies, one from Italy [26] and one from Sweden [28], compared depression and anxiety among patients who underwent $\mathrm{RC}$ with rates in a control group and reported conflicting results. In five studies [26-28, $31,32]$ that also investigated anxiety, reported rates range from 12.5 to $71.3 \%$ after treatment and in one study pre-treatment anxiety was 9.8\% [32] (Table 2).

\section{DISCUSSION}

Distress affects approximately one third of patients undergoing active cancer treatment [33]. Programs that routinely screen for and treat distress were feasible in a multi-disciplinary approach [34]. Distress may include predominantly depression, anxiety, and anger, or is present in a variety of forms [35]. 


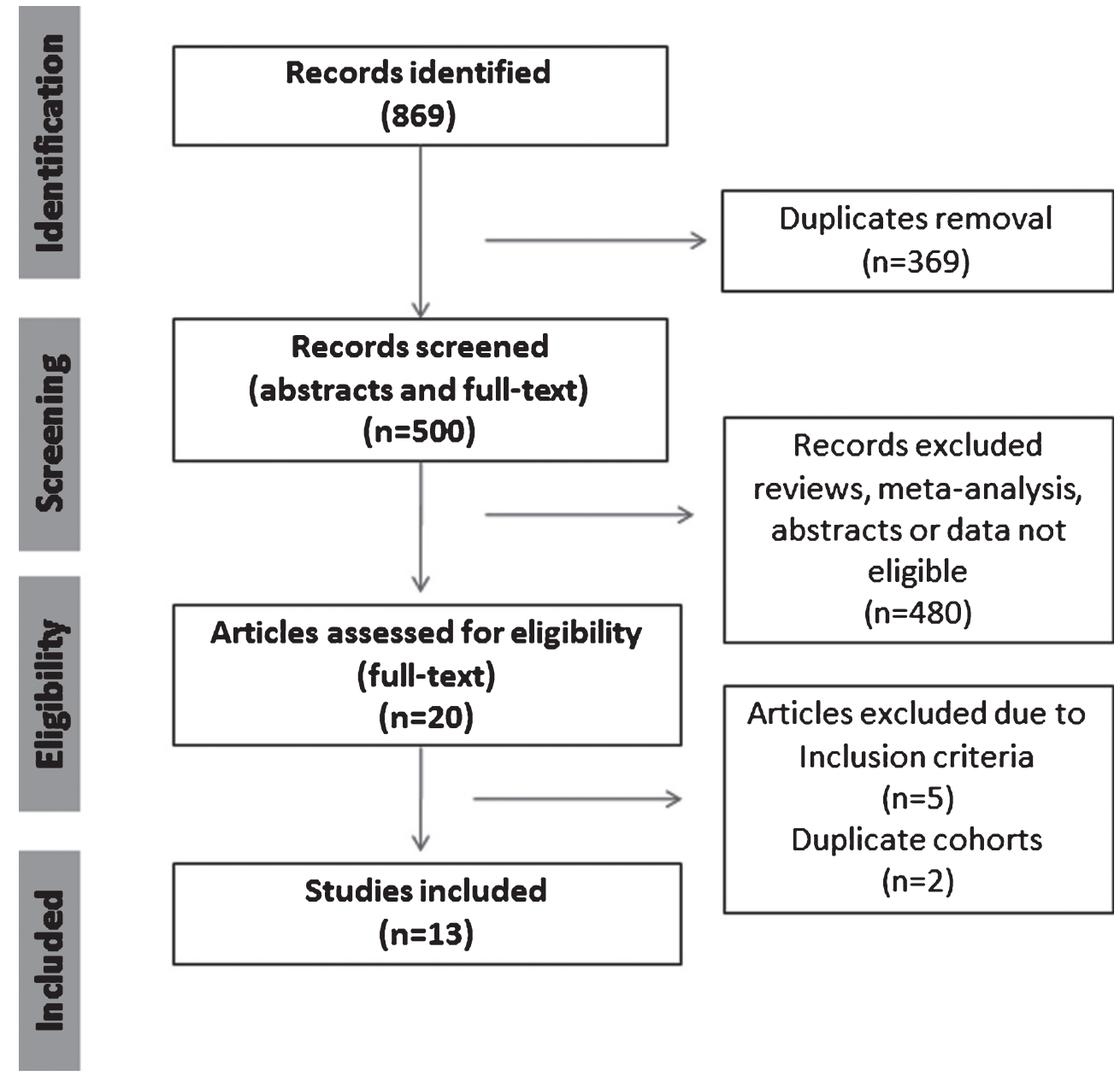

Fig. 1. Summary of evidence search and selection. Selection process for studies to be included is in compliance with PRISMA.

Although evidence shows that willingness to screen for signs/symptoms of these ailments is increasing among cancer professionals, we are still far from increasing adherence [36].

Patients with $\mathrm{BC}$ present at diagnosis with depressive symptoms that might need intervention. Up to $23.1 \%$ of patients with MIBC have clinical relevant depression according to Vakalopoulos et al. study [29]. Another study showed that there is difference in depression rates prior to RC between patients from different countries [22]. On the other hand, Pastore et al. reported lower rates for depression $(5.7 \%)$ and anxiety $(9.8 \%)$ in a cohort of patients that included $88 \%$ of patients with NMIBC [32], suggesting a stage dependent prevalence.

During survivorship depression rates among European patients with $\mathrm{BC}$ ranged from $4.7 \%$ in a Swedish cohort [27] to $11.1 \%$ in Italian one [26]. Moreover, another Swedish study [28] showed no difference in depression compared to controls (26 vs. 37\%) contrary to the Italian study findings [26]. Higher depression symptoms rates were reported among survivors from the US $(21.1 \%)$ and China (78\%) [30,31]. This increased rate of depressive symptoms among Asian population might be due to socio-demographic factors such as different stressors, lack of social support, health risk status and behavioural variables [37]. Furthermore, in a large cohort of Korean patients with $\mathrm{BC}, 8.8 \%$ had major depressive disorder [18]. In comparison with depressive symptoms, anxiety rates were more homogenous in European patients ranging from 12.5 to $23 \%$, contrary, $71.3 \%$ of Chinese patients had anxiety symptoms [31]. The identification, treatment and management of concurrent psychological distress should be a key clinical objective as a means of enhancing 


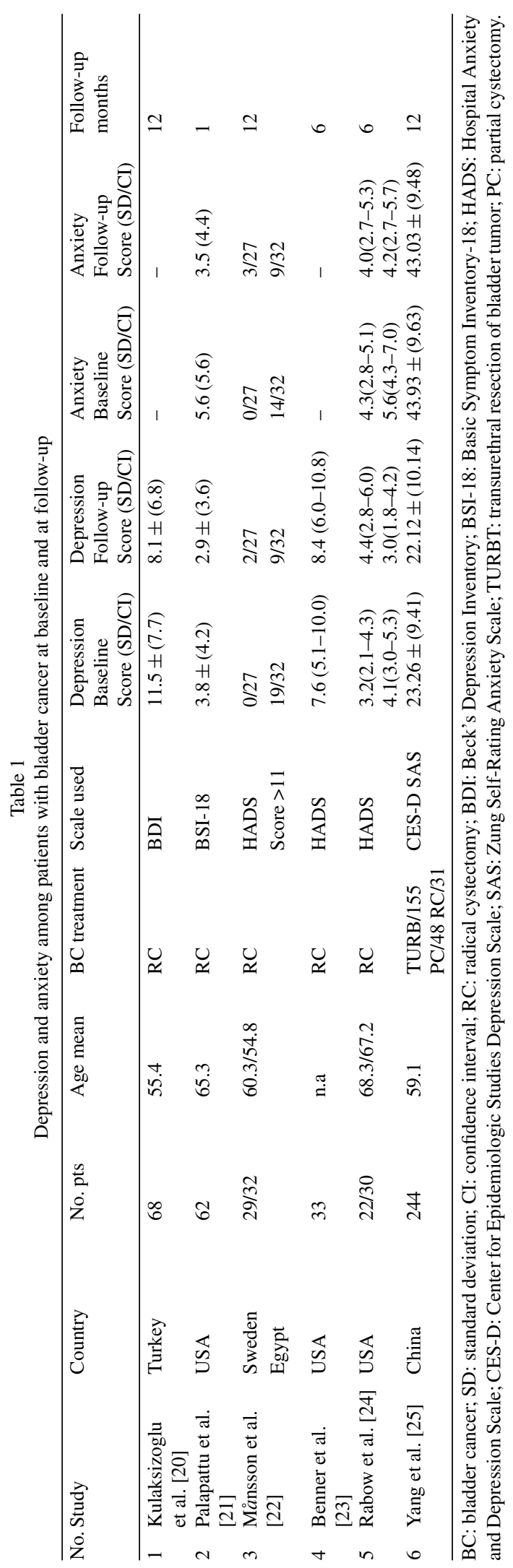

clinical outcomes and patient quality of Life (QoL) [13].

Benner at al. [23] showed that the average HADS scores for depression at 4 and 6 months after RC were at borderline abnormal while initial pre-cystectomy scores were in the normal range, suggesting that there is a negative trend in depression symptoms at 6 months after RC. Furthermore, preoperative depression and anxiety condition had a higher negative impact on increasing symptoms at 6 months. Similarly, in newly diagnosed BC Chinese patients, Yang et al. [25] reported abnormal mean levels of depression scores (CES-D, cut-off 16) that were maintained at 12 months after BC treatment with no intervention to reduce depression. Also, anxiety levels were at borderline normal levels prior and after BC treatment (SAS, cut-off 50). In contrast, when referring patients with clinical pre-treatment depression for specialized treatment, the depression rates decreased from $23 \%$ to $16 \%$ at 12 months after RC [20]. Palapattu et al. [21] showed that at one month after RC, depression $(p<0.034)$ and anxiety $(p<0.0004)$ scores significantly decreased from preoperative to postoperative. Similarly, Månsson et al. showed that significant reduction of depression and anxiety at 12 months in Egyptian patients who underwent RC. Patients who were under palliative care after RC experienced also significant reduction of anxiety and depression than controls who were included in a standard of care program [24]. The true prevalence of psychological morbidity experienced by patients with $\mathrm{BC}$ across the treatment spectrum is poorly understood with many patients being left untreated. Taken all together, one should focus on pre-treatment screening of depression and anxiety. Indeed, recent findings suggested that cancer patients who are suffering from clinical depression and anxiety are less likely to adhere to their treatment plan and are more likely to experience adverse reactions to their treatment [38].

Regarding therapy possibilities, a meta-analysis demonstrated that cognitive behavioural therapy (CBT) offers the highest benefit, particularly for depression [7, 39]. Recently published long-term results of CBT after surgery for early-stage breast cancer revealed that patients who were assigned to therapy had a significantly lower rate of depressive symptoms and better QoL at 15 years compared to controls. The results suggests that early implementation of CBT interventions might improve long-term psychosocial functioning also in other cancers [40].

According to most studies, depression and anxiety symptoms were frequent in patients with $\mathrm{BC}$. 


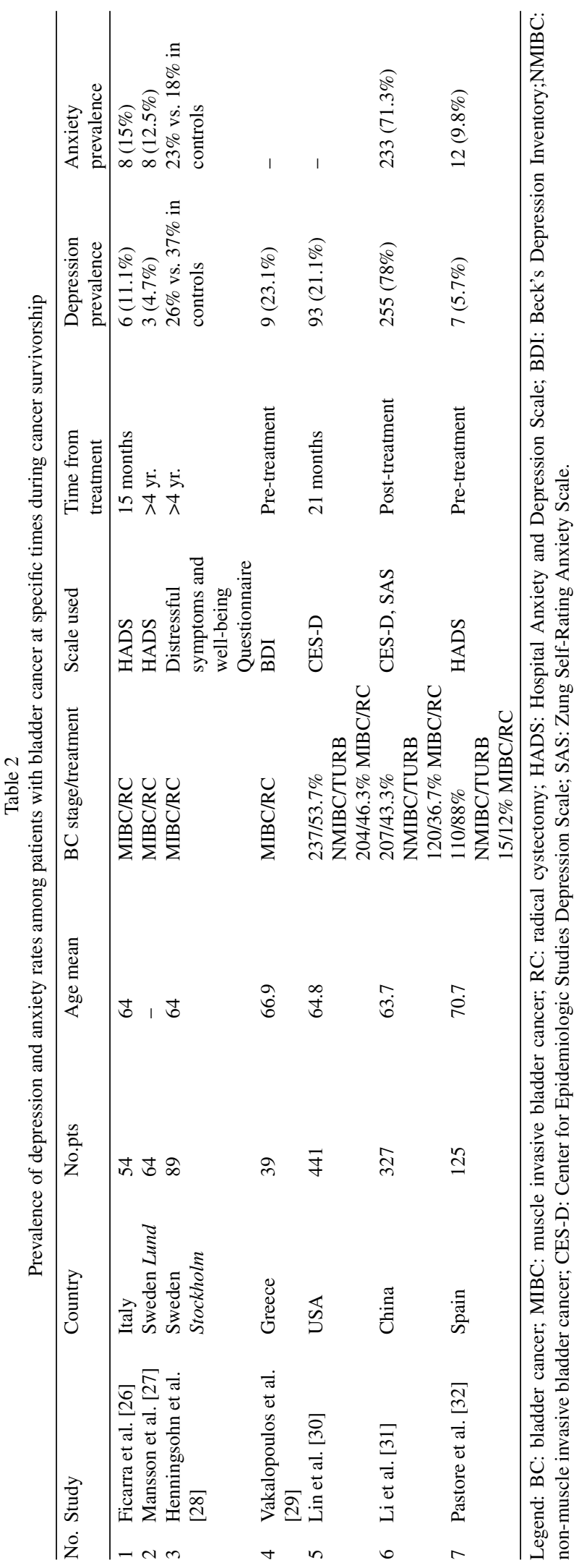

To identify patients who have such psychological comorbidities a routine pre-treatment screening is needed and patients that might have depression and anxiety symptoms should be referred to a psychiatrist/psychologist. Regarding the type of intervention, further studies are needed to assess which type is most beneficial for treating depression and anxiety in patients with $\mathrm{BC}$ as they have specific issues (i.e., body image perception) compared to other cancer patients. Furthermore, geriatric and gender specific research is needed to help better understand and address this unmet need.

Despite a systematic approach, there are several limitations of this systematic review. Firstly, we only reviewed English language literature, and thus possible relevant studies published in other languages were not investigated. Secondly, we did not use formal meta-analytic techniques as a wide range of scales were used to determine depression and anxiety symptoms rates among patients with $\mathrm{BC}$.

\section{CONCLUSION}

Prevalence of depression and anxiety in patients with $\mathrm{BC}$ is high across all cancer stages and status (i.e. pre-treatment to survivorship). Moreover, it is heterogeneous regarding geographical distribute with differences between Caucasians and Asian populations. Treatment with $\mathrm{RC}$ resulted in a reduction of depression and anxiety at 1 year after surgery. Further research is needed to assess depression and anxiety prior and post treatment in the same cohort and to investigate which type of intervention might be beneficial in on at risk-population after pre-treatment screening for depression and anxiety.

\section{ACKNOWLEDGMENTS}

L.V had an Erasmus+ trainee scholarship. M.D.V had a EUSP lab/clinical fellowship awarded by EAU (European Association of Urology) and an Ernst Mach Grant awarded by OeAD, Austria.

\section{REFERENCES}

[1] WHO|Depression. WHO n.d. http://www.who.int/ mediacentre/factsheets/fs369/en/ (accessed March 20, 2018).

[2] Kok RM, Reynolds CF. Management of depression in older adults: A review. JAMA. 2017;317:2114-22. 
[3] Mitchell AJ, Chan M, Bhatti H, Halton M, Grassi L, Johansen C, et al. Prevalence of depression, anxiety, and adjustment disorder in oncological, haematological, and palliative-care settings: A meta-analysis of 94 interviewbased studies. Lancet Oncol. 2011;12:160-74.

[4] Krebber AMH, Buffart LM, Kleijn G, Riepma IC, de Bree $\mathrm{R}$, Leemans CR, et al. Prevalence of depression in cancer patients: A meta-analysis of diagnostic interviews and selfreport instruments. Psychooncology. 2014;23:121-30.

[5] Mitchell AJ, Ferguson DW, Gill J, Paul J, Symonds P. Depression and anxiety in long-term cancer survivors compared with spouses and healthy controls: A systematic review and meta-analysis. Lancet Oncol. 2013;14: 721-32.

[6] Yousaf U, Christensen M-L, Engholm G, Storm HH. Suicides among Danish cancer patients 1971-1999. Br J Cancer. 2005;92:995-1000.

[7] Akechi T, Okuyama T, Onishi J, Morita T, Furukawa TA. Psychotherapy for depression among incurable cancer patients. Cochrane Database Syst Rev. 2008:CD005537.

[8] Meister R, Jansen A, Berger M, Baumeister H, Bschor $\mathrm{T}$, Harfst $\mathrm{T}$, et al. Psychotherapy of depressive disorders: Procedures, evidence and perspectives. Nervenarzt. 2018;89:241-51.

[9] Pruckner N, Holthoff-Detto V. Antidepressant pharmacotherapy in old-age depression-a review and clinical approach. Eur J Clin Pharmacol. 2017;73:661-7.

[10] Watts S, Prescott P, Mason J, McLeod N, Lewith G. Depression and anxiety in ovarian cancer: A systematic review and meta-analysis of prevalence rates. BMJ Open. 2015;5:e007618.

[11] Ferrell BR, Temel JS, Temin S, Alesi ER, Balboni TA, Basch EM, et al. Integration of Palliative Care Into Standard Oncology Care: American Society of Clinical Oncology Clinical Practice Guideline Update. J Clin Oncol Off J Am Soc Clin Oncol. 2017;35:96-112.

[12] Smith CB, Phillips T, Smith TJ. Using the new ASCO clinical practice guideline for palliative care concurrent with oncology care using the TEAM approach. Am Soc Clin Oncol Educ Book Am Soc Clin Oncol Meet. 2017;37:71423.

[13] Watts S, Leydon G, Birch B, Prescott P, Lai L, Eardley S, et al. Depression and anxiety in prostate cancer: A systematic review and meta-analysis of prevalence rates. BMJ Open. 2014;4:e003901.

[14] Smith AB, Rutherford C, Butow P, Olver I, Luckett T, Grimison P, et al. A systematic review of quantitative observational studies investigating psychological distress in testicular cancer survivors. Psychooncology. 2017.

[15] Afshar M, Bardoli AD, Tanner J-R, Macfarlane N, Pickering L, Anderson C, et al. 68 - Patients with urological malignancy are 5 times more likely to commit suicide: A large national cohort study. Abstr EAU18 - 33rd Annu EAU Congr. 2018;17:e100.

[16] Mohamed NE, Chaoprang Herrera P, Hudson S, Revenson TA, Lee CT, Quale DZ, et al. Muscle invasive bladder cancer: Examining survivor burden and unmet needs. J Urol. 2014;191:48-53.

[17] Mohamed NE, Pisipati S, Lee CT, Goltz HH, Latini DM, Gilbert FS, et al. Unmet informational and supportive care needs of patients following cystectomy for bladder cancer based on age, sex, and treatment choices. Urol Oncol. 2016;34:531.e7-531.e14.

[18] Park B, Youn S, Yi K-K, Lee S-Y, Lee J-S, Chung S. The prevalence of depression among patients with the top ten most common cancers in South Korea. Psychiatry Investig. 2017; 14:618-25.

[19] Moher D, Liberati A, Tetzlaff J, Altman DG, PRISMA Group. Preferred reporting items for systematic reviews and meta-analyses: The PRISMA statement. BMJ. 2009;339:b2535.

[20] Kulaksizoglu H, Toktas G, Kulaksizoglu IB, Aglamis E, Unlüer E. When should quality of life be measured after radical cystectomy? Eur Urol. 2002;42:350-5.

[21] Palapattu GS, Haisfield-Wolfe ME, Walker JM, BrintzenhofeSzoc K, Trock B, Zabora J, et al. Assessment of perioperative psychological distress in patients undergoing radical cystectomy for bladder cancer. J Urol. 2004;172:1814-7.

[22] Månsson A, Al Amin M, Malmström P-U, Wijkström H, Abol Enein H, Månsson W. Patient-assessed outcomes in Swedish and Egyptian men undergoing radical cystectomy and orthotopic bladder substitution-a prospective comparative study. Urology. 2007;70:1086-90.

[23] Benner C, Greenberg M, Shepard N, Meng MV, Rabow MW. The natural history of symptoms and distress in patients and families following cystectomy for treatment of muscle invasive bladder cancer. J Urol. 2014;191:937-42.

[24] Rabow MW, Benner C, Shepard N, Meng MV. Concurrent urologic and palliative care after cystectomy for treatment of muscle-invasive bladder cancer. Urol Oncol. 2015;33:267.e23-29.

[25] Yang Y-L, Liu L, Li M-Y, Shi M, Wang L. Psychological disorders and psychosocial resources of patients with newly diagnosed bladder and kidney cancer: A cross-sectional study. Plos One. 2016;11:e0155607.

[26] Ficarra V, Righetti R, D’Amico A, Pilloni S, Balzarro M, Schiavone D, et al. General state of health and psychological well-being in patients after surgery for urological malignant neoplasms. Urol Int. 2000;65:130-4.

[27] Månsson A, Davidsson T, Hunt S, Månsson W. The quality of life in men after radical cystectomy with a continent cutaneous diversion or orthotopic bladder substitution: Is there a difference? BJU Int. 2002;90:386-90.

[28] Henningsohn L, Steven K, Kallestrup EB, Steineck G. Distressful symptoms and well-being after radical cystectomy and orthotopic bladder substitution compared with a matched control population. J Urol. 2002;168:168-74; discussion 174-175.

[29] Vakalopoulos I, Dimitriadis G, Anastasiadis A, Gkotsos G, Radopoulos D. Does intubated ureteroureterocutaneostomy provide better health-related quality of life than orthotopic neobladder in patients after radical cystectomy for invasive bladder cancer? Int Urol Nephrol. 2011;43:743-8.

[30] Lin J, Blalock JA, Chen M, Ye Y, Gu J, Cohen L, et al. Depressive symptoms and short telomere length are associated with increased mortality in bladder cancer patients. Cancer Epidemiol Biomarkers Prev. 2015;24:336-43.

[31] Li M, Wang L. The associations of psychological stress with depressive and anxiety symptoms among chinese bladder and renal cancer patients: The mediating role of resilience. PloS One. 2016;11:e0154729.

[32] Pastore AL, Mir A, Maruccia S, Palleschi G, Carbone A, Lopez C, et al. Psychological distress in patients undergoing surgery for urological cancer: A single centre cross-sectional study. Urol Oncol. 2017.

[33] Carlson LE, Angen M, Cullum J, Goodey E, Koopmans J, Lamont $\mathrm{L}$, et al. High levels of untreated distress and fatigue in cancer patients. Br J Cancer. 2004;90:2297-304. 
[34] Carlson LE, Waller A, Mitchell AJ. Screening for distress and unmet needs in patients with cancer: Review and recommendations. J Clin Oncol Off J Am Soc Clin Oncol. 2012;30:1160-77.

[35] Graves KD, Arnold SM, Love CL, Kirsh KL, Moore PG, Passik SD. Distress screening in a multidisciplinary lung cancer clinic: Prevalence and predictors of clinically significant distress. Lung Cancer Amst Neth. 2007;55:215-24.

[36] Jones LE, Doebbeling CC. Suboptimal depression screening following cancer diagnosis. Gen Hosp Psychiatry. 2007;29:547-54.

[37] Peltzer K, Pengpid S. High prevalence of depressive symptoms in a national sample of adults in Indonesia: Childhood adversity, sociodemographic factors and health risk behaviour. Asian J Psychiatry. 2018;33:52-9.
[38] Caruso R, Nanni MG, Riba M, Sabato S, Mitchell AJ, Croce E, et al. Depressive spectrum disorders in cancer: Prevalence, risk factors and screening for depression: A critical review. Acta Oncol Stockh Swed. 2017;56:146-55.

[39] Okuyama T, Akechi T, Mackenzie L, Furukawa TA. Psychotherapy for depression among advanced, incurable cancer patients: A systematic review and meta-analysis. Cancer Treat Rev. 2017;56:16-27.

[40] Stagl JM, Bouchard LC, Lechner SC, Blomberg BB, Gudenkauf LM, Jutagir DR, et al. Long-term psychological benefits of cognitive-behavioral stress management for women with breast cancer: 11-year follow-up of a randomized controlled trial. Cancer. 2015;121:1873-81. 